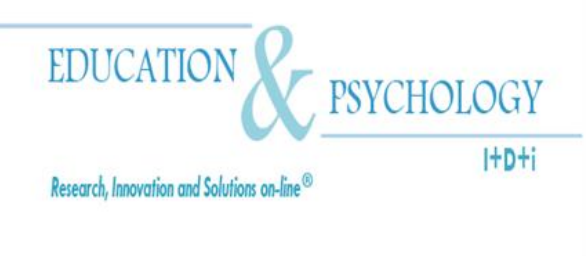

\title{
Sexismo y agresiones físicas, sexuales y verbales-emocionales, en relaciones de noviazgo de estudiantes universitarios
}

\author{
José Luis Rojas-Solís ${ }^{1}$, Eugenio Carpintero Raimúndez ${ }^{2}$ \\ ${ }^{1}$ y 2 Departamento de Psicología Evolutiva y de la Educación, \\ Universidad de Salamanca, Salamanca.
}

\section{España}

Correspondencia: José Luis Rojas-Solís. Departamento de Psicología Evolutiva y de la Educación, Facultad de Psicología, Universidad de Salamanca. Avda. de la Merced, 109-131. 37005. Salamanca, España. E-mail: 1uisrojas@usal.es

(C) Education \& Psychology I+D+i and Editorial EOS (Spain) 


\section{Resumen}

Introducción. En los últimos años el interés sobre la violencia en relaciones de pareja se ha desplazado hacia poblaciones más jóvenes. Diferentes modelos explicativos asocian diversos factores a este fenómeno. Sin embargo desde la teoría feminista se otorga un gran peso a las actitudes sexistas en la aparición y mantenimiento de conductas violentas. El objetivo de este estudio consiste en comprobar empíricamente la relación teórica entre estas variables para diseñar planes específicos de prevención e intervención sobre este problema.

Método. Se analizan los niveles de sexismo benevolente y hostil hacia la mujer; la frecuencia de agresiones físicas, sexuales y verbales-emocionales, perpetradas y sufridas; y la relación entre estas variables en una muestra de 453 alumnos de diferentes titulaciones de la Universidad de Salamanca, con edades entre los 18 y 36 años. La selección de la muestra fue estratificada y no probabilística, el número de miembros para cada estrato se determinó por asignación proporcional y el criterio de inclusión fue tener o haber tenido una relación de pareja.

Resultados. Se hallaron diferencias significativas en los niveles de sexismo entre hombres y mujeres. Los hombres manifestaron haber perpetrado más agresiones sexuales, las mujeres más agresiones verbales-emocionales y no hubo diferencias significativas en la perpetración de agresiones físicas. Los hombres indicaron una mayor frecuencia en las agresiones físicas sufridas, mientras que no se hallaron diferencias significativas entre sexos en cuanto a agresiones sexuales y verbales-emocionales sufridas. Por último, no se obtuvieron fuertes correlaciones entre actitudes sexistas y comportamientos agresivos cometidos.

Discusión y Conclusión. Los resultados obtenidos destacan la necesidad de investigar sobre el sexismo ambivalente hacia los hombres así como la conveniencia de más estudios cuantitativos y cualitativos acerca de la violencia bidireccional en las relaciones de noviazgo de jóvenes, lo que a su vez sugeriría la adopción de posturas ecológicas o modelos inclusivos que en su explicación conlleven multifactorialidad y alternativas a la dicotomía agresor-víctima para el entendimiento, prevención e intervención en la violencia en parejas jóvenes.

Palabras Clave: sexismo, agresiones, noviazgo, universitarios

Recibido: $21 / 02 / 11$

Aceptación inicial: 25/02/11

Aceptación final: 30/05/11 


\title{
Sexism and physical, sexual and verbal-emotional aggression in courtship relationships in university students
}

\begin{abstract}
Introduction. Recently research on partner violence has shifted from adults to younger populations. Different models have explained this phenomenon trough out many factors. However feminist theory has given great weight to the sexist attitudes in the development and maintenance of violent behavior. The aim of this study is corroborate empirically the theoretical relationship between these variables to devise prevention and intervention plans for the eradication of this problem.
\end{abstract}

Method. We analyzed the levels of benevolent and hostile sexism toward women, the frequency of physical, sexual and verbal, emotional aggressions, perpetrated and suffered, and the relationship between these variables in a sample of 453 students in different degrees from the University of Salamanca, aged between 18 and 36 years. The sample selection was stratified and not random; the number of members for each stratum was determined by proportional allocation and the inclusion criterion was having or having had a relationship.

Results. The results indicate significant differences in levels of sexism among men and women. Males reported having perpetrated significantly more sexual aggressions, females reported having perpetrated significantly more verbal-emotional aggressions, but no sex differences were found in the frequency of commited physical aggressions. About the frequency of suffered aggressions, men reported a higher frequency in physical aggressions and there were no significant differences by sex in the suffered sexual and verbal-emotional aggressions. No strong links were observed between sexist attitudes and aggressive behaviors.

Discussion and Conclusion: Results highlight the need for research on ambivalent sexism toward men and the desirability of more quantitative and qualitative studies on bidirectional violence in dating relationships of young people, which in turn would suggest the ecological or posturing inclusive models in their explanation involving multiple factors and alternatives to the abuser-victim dichotomy for understanding, prevention and intervention in violence in young couples.

Keywords: sexism, agressions, dating relationship, university students. 


\section{Introducción}

La identificación de la violencia en parejas como problema de salud pública ha representado un avance social. Sin embargo sus características han significado un verdadero reto conceptual, metodológico e ideológico porque dependiendo de la perspectiva teórica o ideológica adoptada se priorizarán factores individuales, estructurales o culturales (Hattery, 2009). Por ello, en la literatura científica se pueden encontrar dos grandes planteamientos sobre el fenómeno con sus respectivas implicaciones teóricas y metodológicas: el unidireccional, que basado en teorías feministas contempla la violencia contra la mujer como el resultado de una sociedad patriarcal donde el varón ha tenido el papel predominante y ha ejercido el poder y la violencia contra ella (Pelegrín y Garcés, 2004), y la propuesta del modelo bidireccional donde ambos sexos pueden ser tanto agresores como víctimas (Archer, 2000).

A la par de ello, en los últimos años, la atención sobre la violencia se ha desplazado hacia parejas cada vez más jóvenes por diversas razones (Póo y Vizcarra, 2008): su alarmante tasa de prevalencia, las consecuencias físicas y mentales en las víctimas o su aparición en una etapa del ciclo vital cuando comienzan las relaciones románticas y se aprenden pautas de interacción que al extenderse a la edad adulta pueden convertirse en precursores de una violencia mucho más grave (González-Ortega, Echeburúa y Corral, 2008). Pese a todo esto, en España, el número de investigaciones sobre esta población aún sigue siendo restringido (Fernández-Fuertes y Fuertes, 2010).

Debido a que la perspectiva feminista pretende contribuir a la visibilidad de los problemas de salud pública diferenciando las condiciones estructurales y culturales en que viven hombres y mujeres, esta investigación se concentra en el estudio de las agresiones en relaciones de noviazgo de universitarios incluyendo la variable del sexismo por su carácter de factor de riesgo en la aparición de conductas violentas contra la mujer (Expósito y Herrera, 2009), su presencia en propuestas de prevención e intervención educativa y psicosocial (Pozo, Martos y Alonso, 2010; Ruiz, Expósito y Bonache, 2010) y por la conveniencia de la corroboración empírica de su influencia en la perpetración de agresiones contra las mujeres (GarcíaLeiva, Palacios, Torrico y Navarro, 2007).

En el contexto internacional, el sexismo también ha sido relacionado con la agresión hacia las mujeres. Por ejemplo Begany y Milburn (2002) señalan la relación existente entre el sexismo hostil y actitudes autoritarias como predictoras de acoso sexual. Chapleau, Oswald y 
Russell (2007), con una muestra de alumnado universitario, hallaron una correlación positiva en los hombres entre sexismo hostil y aceptación de la agresión sexual; en el caso de las mujeres hallaron una alta correlación entre un maternalismo y la aceptación de la agresión. Por otra parte Krahé, Bieneck y Scheinberger-Olwig (2007) enfatizan en su trabajo la estrecha relación entre los guiones sexuales y la agresión sexual hacia la mujer. En España, la literatura sobre violencia de género también considera al sexismo como un factor de riesgo en la violencia contra la mujer (Ferrer y Bosch, 2005), se le relaciona con la discriminación (Pozo, Alonso, Hernández y Mellado, 2005), el maltrato a las mujeres (Torrico et al., 2005), la aceptación de la violencia de género (Expósito y Moya, 2005) e incluso se le otorga un carácter de predictor de la violencia contra la mujer (García-Leiva et al., 2007; Palacios et al., 2005). Sin embargo la demostración empírica de algunas de estas relaciones hasta ahora no ha sido muy contundente (Murnen, Wright y Kaluzny, 2002; Santana, Raj, Decker, La Marche y Silverman, 2006).

Por ello en el presente estudio se utilizan conceptos provenientes de la Teoría del sexismo ambivalente de Glick y Fiske (1996) en la que se identifica al sexismo ambivalente como la combinación de actitudes sexistas con sentimientos subjetivamente negativos (hostiles) y positivos (benevolentes) hacia el sexo femenino o masculino. Hasta el momento en que se llevó a cabo la revisión teórica sobre la evaluación de sexismo en población española se halló sólo un instrumento contextualizado para adolescentes y jóvenes: la Escala de Detección de Sexismo en Adolescentes (Recio, Cuadrado y Ramos, 2007) elaborada para edades un poco distintas a la de universitarios, pero cuyos ítems no están contrapuestos al contexto y quehacer cotidiano de la muestra de este estudio.

En cuanto a la medición de la violencia en parejas jóvenes el panorama relativo a instrumentos también fue limitado debido a las pocas alternativas existentes y a que en su mayoría son adaptaciones de instrumentos para evaluar la violencia en adultos y otros contextos. Después de la revisión del estado de la cuestión las opciones recayeron entre la versión modificada de la Escala de solución de conflictos (MCTS, Muñoz-Rivas, Graña, O’Leary y González, 2007a) o el Conflict in Adolescent Dating Relationships Inventory (CADRI, FernándezFuertes, Fuertes y Pulido, 2006). Se eligió el segundo instrumento debido a que el tipo de preguntas y las formas de agresiones que evalúa son muy cercanas a la realidad de las relaciones de pareja de jóvenes universitarios; por la naturaleza del instrumento seleccionado el planteamiento de la violencia no fue unidireccional. 


\section{Objetivos e hipótesis}

Los objetivos de este trabajo son: 1) analizar los niveles de sexismo benevolente y hostil en la muestra de hombres y mujeres, 2) la frecuencia de agresiones físicas, sexuales y verbales-emocionales tanto perpetradas como sufridas por ambos sexos y 3) hallar la relación entre niveles de sexismos y frecuencias de agresiones.

Se esperaba encontrar en los hombres un mayor nivel de sexismo hostil (Ferrer y Bosch, 2000), una mayor frecuencia en la perpetración de agresiones físicas (Toldos, 2005) y sexuales y se confirmaría que un nivel alto de sexismo hostil estaría fuerte y positivamente asociado con la perpetración de agresiones sexuales (Chapleau et al. 2007) y físicas (Ferrer, Bosch, Ramis, Torres y Navarro, 2006). Por su parte, las mujeres mostrarían un mayor nivel de sexismo benevolente y mayor frecuencia en la perpetración de agresiones verbalesemocionales (Alonso, Lobato y Rodríguez, 2007).

\section{Método}

\section{Participantes}

La selección de la muestra fue estratificada, se dividió la población en ramas de estudio y se determinó el número de miembros para cada estrato por asignación proporcional; el criterio de inclusión en el estudio fue tener o haber tenido una relación de pareja, en este segundo caso se solicitaba responder al cuestionario de acuerdo a la relación más larga que se había tenido; la media de duración de la relación actual en meses fue $\mathrm{M}=31.19$ (DT = 27.66) y la relación pasada $\mathrm{M}=14.68$ ( $\mathrm{DT}=14.15)$. La muestra final fue constituida por 453 sujetos (74.8\% mujeres y 25.2\% hombres), alumnos de grado de la Universidad de Salamanca, con edades comprendidas entre 18 y 36 años $(M=21.44$, DT $=2.42)$. El $15 \%$ de la muestra cursaba estudios en las ramas de Enseñanzas Técnicas, el $25 \%$ en Ciencias Experimentales y de la Salud, un $25 \%$ en Humanidades y un $35 \%$ en estudios comprendidos en las Ciencias Sociales y Jurídicas. Al momento del estudio el $52.6 \%$ de hombres y $57 \%$ de mujeres tenían una relación de noviazgo y el 44\% del total de la muestra describió una relación pasada. 


\section{Instrumentos y variables}

El instrumento utilizado constó de tres secciones. En la primera se formularon preguntas relativas a las características generales de la muestra, posteriormente se incluyeron cuestionarios para medir niveles de sexismo y frecuencia de las agresiones.

La Escala de Detección de Sexismo en Adolescentes (DSA; Recio et al., 2007) está adecuada a la teoría del sexismo ambivalente y tiene validez convergente con otras escalas elaboradas para el mismo fin (Expósito, Moya y Glick, 1998; Glick y Fiske, 1996). Se constituye por 26 ítems, de los cuales 16 evalúan el sexismo hostil (ejemplo: "Las mujeres son manipuladoras por naturaleza") y 10, el sexismo benevolente (ejemplo: "Las mujeres son, por naturaleza, más pacientes y tolerantes que los hombres”). La escala de respuesta es tipo Likert con 6 anclajes donde 1 significa "Totalmente en desacuerdo" y 6 "Totalmente de acuerdo"; a mayor puntuación, mayor sexismo.

Las dos formas de sexismo se definieron de la siguiente forma: 1) el sexismo hostil es un prejuicio hacia las mujeres que las considera en una situación de inferioridad con respecto a los hombres; mientras que 2) el sexismo benevolente es un tipo de prejuicio hacia las mujeres basado en una visión estereotipada y limitada de la mujer, con un tono afectivo-positivo, unido a conductas de apoyo, al mismo tiempo que se enfatiza su debilidad y necesidad de protección.

El Conflict in Adolescent Dating Relationships Inventory (CADRI; Fernández-Fuertes, Fuertes y Orgaz, 2008), en una versión revisada del CADRI original (Fernández-Fuertes et al., 2006; Wolfe et al., 2001) con cinco subescalas que miden la frecuencia de diferentes tipos de agresiones; las preguntas son bidireccionales (víctima/agresor-a) con una escala de respuesta tipo Likert donde $0=$ "Nunca", 1 = "Rara" vez, 2 = "A veces" y 3 = "Con frecuencia." Se utilizaron las subescalas referidas a las agresiones sexuales cometidas y sufridas evaluadas por 6 ítems (ejemplo: "Le/Me besé/o cuando él/ella/yo no quería), las agresiones físicas cometidas y sufridas evaluadas por 6 ítems (ejemplo: “Le/Me lancé/zó algún objeto) y las agresiones verbales-emocionales cometidas, evaluadas por 10 ítems (ejemplo: "Le/Me amenacé/zó con dejar la relación). 
Las tres formas de agresiones se definieron así: 1) las agresiones verbalesemocionales se refieren al uso de la palabra para hacer sentir a una persona que no hace nada bien, ridiculizarla, insultarla o humillarla, ya sea en la intimidad o ante familiares, amigos o desconocidos; 2) las agresiones físicas son aquellas conductas que, cuando se realizan, tienen el potencial de ocasionar algún daño físico a otro sujeto, independientemente del alcance real de la lesión; y 3) las agresiones sexuales son aquellos contactos de índole sexual no consentidos.

\section{Procedimiento}

La administración del cuestionario se realizó de forma colectiva en diversas Facultades de la Universidad de Salamanca con una duración aproximada de 40 minutos y bajo la supervisión de un miembro del equipo de investigación. Después de explicar los objetivos generales del estudio, se hizo énfasis en el carácter voluntario y confidencial de la participación. Los directores de los centros y los profesores facilitaron el acceso a la muestra, así como los horarios de clase y aulas para que el alumnado pudiera contestar el cuestionario y, una vez cumplimentado éste, se informó a los participantes de los servicios que tiene la universidad a disposición del estudiantado en cuestiones relacionadas con problemáticas psicológicas, relacionales y sexuales.

\section{Análisis de datos}

Las primeras técnicas estadísticas utilizadas fueron los análisis de fiabilidad de las escalas utilizadas a través del alfa de Cronbach además de los análisis descriptivos de las variables. Con la finalidad de establecer comparaciones entre hombres y mujeres se llevó a cabo la prueba U de Mann-Whitney debido a que las características de la muestra no cumplían con los supuestos necesarios para utilizar estadística paramétrica. Por último, para constatar la magnitud de la asociación y dirección de la relación entre las variables se utilizó el coeficiente de correlación de Spearman. Los análisis estadísticos se realizaron con el programa informático SPSS 15.0 para Windows.

\section{Resultados}

\section{Consistencia interna}

En primer lugar se determinó la consistencia interna de las subescalas empleadas a través del alfa de Cronbach encontrándose niveles muy aceptables en las subescalas de sexis- 
mo hostil (.87) y benevolente (.84) y la de agresiones verbales-emocionales cometidas (.79) y sufridas (80); resultados que contrastan con los índices de las subescalas de agresiones físicas cometidas (.58) y sufridas (.62), así como de la subescalas de agresiones sexuales cometidas (.57) y sufridas (.57).

Las puntuaciones medias de las subescalas de sexismo en el total de la muestra fueron: sexismo hostil $\mathrm{M}=1.36(\mathrm{DT}=.49)$ y sexismo benevolente, $\mathrm{M}=2.57(\mathrm{DT}=.90)$. En cuanto a las diferencias de puntuaciones entre hombres y mujeres, los resultados de la prueba de $\mathrm{U}$ de Mann-Whitney revelaron diferencias significativas entre sexismo hostil $(\mathrm{U}=14367 ; \mathrm{Z}=$ 4.138; $\mathrm{p}=.00)$ manifestado por hombres $(\mathrm{M}=1.55, \mathrm{DT}=0.60)$ y mujeres $(\mathrm{M}=1.30, \mathrm{DT}=$ $0.44)$ y en las puntuaciones en el sexismo benevolente $(U=16702.5 ; Z=-2.169 ; p=.03) \mathrm{de}$ hombres $(\mathrm{M}=2.39$, DT $=0.90)$ y mujeres $(\mathrm{M}=2.62, \mathrm{DT}=0.90)$.

\section{Agresiones}

En cuanto a los resultados de las agresiones físicas, en la tabla 1 se puede observar que casi la totalidad de las respuestas de hombres y mujeres en la subescala de agresiones físicas cometidas se concentraron en la opción "nunca" y no se halló diferencia significativa (U = $18461 ; \mathrm{Z}=-1.185 ; \mathrm{p}=.236)$ entre las puntuaciones medias de hombres $(\mathrm{M}=0.03$, $\mathrm{DT}=$ $0.14)$ y mujeres $(\mathrm{M}=0.04$, DT $=0.12)$. Sin embargo en las agresiones físicas sufridas la media en el grupo de los hombres $(\mathrm{M}=0.08$, $\mathrm{DT}=0.22)$ fue significativamente superior $(\mathrm{U}=$ 17746; $\mathrm{Z}=-2.398 ; \mathrm{p}=.016)$ a la de las mujeres $(\mathrm{M}=0.02$, DT $=0.09)$. La proporción de hombres que se declararon víctimas de agresiones por parte de su pareja fue mayor que la de las mujeres; las agresiones más severas por ejemplo: ítems 30 o 33 fueron menos comunes, pero incluso allí hubo una tendencia similar. 
Tabla 1. Frecuencia de agresiones físicas cometidas y sufridas (Chicos $n=114$; Chicas $n=339$ )

\begin{tabular}{|c|c|c|c|c|c|c|c|c|}
\hline \multicolumn{9}{|c|}{ Agresiones físicas cometidas } \\
\hline \multirow[t]{2}{*}{ Ítem } & \multicolumn{2}{|c|}{ Nunca } & \multicolumn{2}{|c|}{$\begin{array}{c}\text { Rara vez } \\
\text { (entre } 1 \text { o } 2 \\
\text { veces) }\end{array}$} & \multicolumn{2}{|c|}{$\begin{array}{c}\text { A veces } \\
\text { (entre } 3 \text { y } 5 \\
\text { veces) }\end{array}$} & \multicolumn{2}{|c|}{$\begin{array}{l}\text { Con frecuencia } \\
\text { (6 o más veces) }\end{array}$} \\
\hline & $\begin{array}{c}\text { Chicos } \\
\%\end{array}$ & $\begin{array}{c}\text { Chicas } \\
\%\end{array}$ & $\begin{array}{c}\text { Chicos } \\
\%\end{array}$ & $\begin{array}{c}\text { Chicas } \\
\%\end{array}$ & $\begin{array}{c}\text { Chicos } \\
\%\end{array}$ & $\begin{array}{c}\text { Chicas } \\
\%\end{array}$ & $\begin{array}{c}\text { Chicos } \\
\%\end{array}$ & $\begin{array}{c}\text { Chicas } \\
\%\end{array}$ \\
\hline 6 & 98,2 & 92 & 1,8 & 5,9 & 0 & 1,2 & 0 & 0 \\
\hline 22 & 97,4 & 95 & 1,8 & 3,2 & 0,9 & 1,2 & 0 & 0 \\
\hline 26 & 98,2 & 96,8 & 0,9 & 2,7 & 0,9 & 0 & 0 & 0 \\
\hline 28 & 92,1 & 92 & 5,3 & 6,2 & 1,8 & 0,6 & 0,9 & 0,3 \\
\hline 30 & 98,2 & 99,1 & 0,9 & 0,9 & 0 & 0 & 0 & 0 \\
\hline 33 & 99,1 & 99,4 & 0,9 & 0,3 & 0 & 0 & 0 & 0 \\
\hline \multicolumn{9}{|c|}{ Agresiones físicas sufridas } \\
\hline 6 & 92,1 & 97,1 & 5,3 & 2,1 & 2,6 & 0,3 & 0 & 0 \\
\hline 22 & 88,6 & 96,8 & 8,8 & 2,1 & 1,8 & 0,9 & 0,9 & 0,3 \\
\hline 26 & 91,2 & 97,9 & 7 & 1,8 & 1,8 & 0 & 0 & 0 \\
\hline 28 & 91,2 & 94,4 & 7 & 4,4 & 0,9 & 0,6 & 0,9 & 0 \\
\hline 30 & 98,2 & 99,7 & 0,9 & 0,3 & 0 & 0 & 0 & 0 \\
\hline 33 & 99,1 & 99,4 & 0,9 & 0,6 & 0 & 0 & 0 & 0 \\
\hline
\end{tabular}

Con respecto a las agresiones sexuales cometidas, la media de las puntuaciones de los hombres $(M=0.21$, DT $=0.34)$ fue significativamente superior $(U=17070.5 ; Z=-2.052 ; \mathrm{p}=$ $.04)$ a la de las mujeres $(\mathrm{M}=0.13, \mathrm{DT}=0.19)$, pero en las agresiones sexuales sufridas no hubo diferencia significativa $(U=18950 ; Z=-.337 ; p=.736)$ entre hombres $(M=0.19$, DT $=$ $0.30)$ y mujeres $(\mathrm{M}=0.17$, DT $=0.24)$. Como se puede constatar en la tabla 2 , hay una mayor inclinación en los hombres a involucrarse en la perpetración de este tipo de agresiones. En el caso de las agresiones sexuales sufridas la tendencia general de las respuestas fue hacia la opción "nunca", no obstante se puede observar una distribución muy equiparada en las respuestas de ambos sexos con excepción del ítem 2. 
Tabla 2. Frecuencia de agresiones sexuales cometidas y sufridas (Chicos $n=114$; Chicas $n=339$ )

\begin{tabular}{|c|c|c|c|c|c|c|c|c|}
\hline \multicolumn{9}{|c|}{ Agresiones sexuales cometidas } \\
\hline \multirow[t]{2}{*}{ Ítem } & \multicolumn{2}{|c|}{ Nunca } & \multicolumn{2}{|c|}{$\begin{array}{c}\text { Rara vez } \\
\text { (entre } 1 \text { o } 2 \\
\text { veces) }\end{array}$} & \multicolumn{2}{|c|}{$\begin{array}{c}\text { A veces } \\
\text { (entre } 3 \text { y } 5 \\
\text { veces) }\end{array}$} & \multicolumn{2}{|c|}{$\begin{array}{l}\text { Con frecuencia } \\
\text { (6 o más veces) }\end{array}$} \\
\hline & $\begin{array}{c}\text { Chicos } \\
\%\end{array}$ & $\begin{array}{c}\text { Chicas } \\
\%\end{array}$ & $\begin{array}{c}\text { Chicos } \\
\%\end{array}$ & $\begin{array}{c}\text { Chicas } \\
\%\end{array}$ & $\begin{array}{c}\text { Chicos } \\
\%\end{array}$ & $\begin{array}{c}\text { Chicas } \\
\%\end{array}$ & $\begin{array}{c}\text { Chicos } \\
\%\end{array}$ & $\begin{array}{c}\text { Chicas } \\
\%\end{array}$ \\
\hline 2 & 67,5 & 81,7 & 22,8 & 12,1 & 5,3 & 3,8 & 4,4 & 1,2 \\
\hline 11 & 94,7 & 99,1 & 3,5 & 0,3 & 1,8 & 0 & 0 & 0 \\
\hline 13 & 96,5 & 98,5 & 2,6 & 0,6 & 0 & 0 & 0 & 0 \\
\hline 17 & 60,5 & 67,8 & 22,8 & 23,6 & 13,2 & 6,5 & 3,5 & 1,8 \\
\hline 29 & 96,5 & 99,4 & 0,9 & 0,3 & 2,6 & 0 & 0 & 0 \\
\hline 31 & 87,7 & 97,6 & 10,5 & 1,8 & 0,9 & 0,3 & 0 & 0 \\
\hline \multicolumn{9}{|c|}{ Agresiones sexuales sufridas } \\
\hline 2 & 70,2 & 74 & 21,9 & 16,5 & 5,3 & 5,9 & 2,6 & 2,4 \\
\hline 11 & 95,6 & 94,7 & 4,4 & 4,1 & 0 & 0,9 & 0 & 0 \\
\hline 13 & 95,6 & 99,4 & 3,5 & 0,3 & 0 & 0,3 & 0 & 0 \\
\hline 17 & 61,4 & 62,4 & 23,7 & 25,7 & 12,3 & 9,3 & 2,6 & 2,7 \\
\hline 29 & 97,4 & 98,8 & 1,8 & 1,2 & 0,9 & 0 & 0 & 0 \\
\hline 31 & 93 & 93,8 & 4,4 & 5,3 & 0,9 & 0,6 & 0,9 & 0 \\
\hline \multicolumn{9}{|c|}{$\begin{array}{l}\text { 2. Acaricié (ó) sus (mis) pechos, genitales y/o nalgas cuando él/ella (yo) no quería } \\
\text { 11. Le (Me) forcé (ó) a practicar alguna actividad sexual cuando él/ella (yo) no quería } \\
\text { 13. Le (Me) amenacé (ó) para que no se (me) negase a mantener algún tipo de relación } \\
\text { sexual conmigo (con él/ella) } \\
\text { 17. Le (Me) besé (ó) cuando él/ella (yo) no quería } \\
\text { 29. Le (Me) forcé (ó) a tocar mis (sus) pechos, genitales y/o nalgas cuando él/ella (yo) no } \\
\text { quería } \\
\text { 31. Seguí (Siguió) desnudándole/la (me) aunque sabía que él/ella (yo) no quería }\end{array}$} \\
\hline
\end{tabular}

En las agresiones verbales-emocionales cometidas se halló diferencia significativa (U $=16641 ; \mathrm{Z}=-2.224 ; \mathrm{p}=.026)$ entre las puntuaciones de hombres $(\mathrm{M}=0.66, \mathrm{DT}=0.48) \mathrm{y}$ mujeres $(\mathrm{M}=0.75 ; \mathrm{DT}=0.46)$ y no se estableció diferencia significativa $(\mathrm{U}=18602.5 ; \mathrm{Z}=-$ $.597 ; \mathrm{p}=.55)$ entre las agresiones verbales-emocionales sufridas por hombres $(\mathrm{M}=0.73$, DT $=0.50)$ y por mujeres $(\mathrm{M}=0.69, \mathrm{DT}=0.49)$. La distribución y frecuencias de respuesta $\mathrm{a}$ cada ítem se puede observar en la Tabla 3. 
Tabla 3. Frecuencia de agresiones verbales-emocionales cometidas y sufridas (Chicos $n=114$; Chicas $n=339$ )

\begin{tabular}{|c|c|c|c|c|c|c|c|c|}
\hline \multicolumn{9}{|c|}{ Agresiones verbales-emocionales cometidas } \\
\hline \multirow[t]{2}{*}{ Ítem } & \multicolumn{2}{|c|}{ Nunca } & \multicolumn{2}{|c|}{$\begin{array}{c}\text { Rara vez } \\
\text { (entre } 1 \text { o } 2 \\
\text { veces) }\end{array}$} & \multicolumn{2}{|c|}{$\begin{array}{c}\text { A veces } \\
\text { (entre } 3 \text { y } 5 \\
\text { veces) }\end{array}$} & \multicolumn{2}{|c|}{$\begin{array}{l}\text { Con frecuencia } \\
\text { (6 o más veces) }\end{array}$} \\
\hline & $\begin{array}{c}\text { Chicos } \\
\%\end{array}$ & $\begin{array}{c}\text { Chicas } \\
\%\end{array}$ & $\begin{array}{c}\text { Chicos } \\
\%\end{array}$ & $\begin{array}{c}\text { Chicas } \\
\%\end{array}$ & $\begin{array}{c}\text { Chicos } \\
\%\end{array}$ & $\begin{array}{c}\text { Chicas } \\
\%\end{array}$ & $\begin{array}{c}\text { Chicos } \\
\%\end{array}$ & $\begin{array}{c}\text { Chicas } \\
\%\end{array}$ \\
\hline 3 & 46,5 & 40,4 & 33,3 & 37,8 & 15,8 & 18,3 & 4,4 & 2,7 \\
\hline 5 & 29,8 & 23,9 & 28,1 & 32,4 & 28,9 & 33,6 & 13,2 & 9,4 \\
\hline 7 & 42,1 & 39,8 & 36,8 & 36,9 & 16,7 & 19,5 & 4,4 & 2,9 \\
\hline 10 & 43 & 31,9 & 31,6 & 31 & 19,3 & 30,1 & 5,3 & 6,5 \\
\hline 15 & 80,7 & 69,3 & 12,3 & 20,9 & 4,4 & 7,4 & 2,6 & 1,8 \\
\hline 18 & 81,6 & 85 & 15,8 & 11,2 & 1,8 & 3,2 & 0,9 & 0,3 \\
\hline 20 & 93 & 90 & 6,1 & 8,8 & 0,9 & 0,9 & 0 & 0 \\
\hline 21 & 32,5 & 23,6 & 30,7 & 28,6 & 28,1 & 38,9 & 8,8 & 8,3 \\
\hline 25 & 62,3 & 58,1 & 26,3 & 22,1 & 7 & 13,9 & 4,4 & 5,3 \\
\hline 27 & 61,4 & 54,3 & 19,3 & 27,1 & 16,7 & 15 & 2,6 & 2,9 \\
\hline \multicolumn{9}{|c|}{ Agresiones verbales-emocionales sufridas } \\
\hline 3 & 39,5 & 45,7 & 36 & 32,2 & 15,8 & 16,5 & 7,9 & 3,8 \\
\hline 5 & 28,1 & 32,7 & 30,7 & 33,3 & 28,9 & 24,8 & 12,3 & 8 \\
\hline 7 & 43 & 41 & 32,5 & 36,9 & 18,4 & 18,9 & 6,1 & 2,7 \\
\hline 10 & 41,2 & 35,4 & 34,2 & 36,6 & 19,3 & 21,5 & 4,4 & 5,9 \\
\hline 15 & 78,9 & 74,9 & 14,9 & 16,2 & 3,5 & 8,3 & 2,6 & 0,6 \\
\hline 18 & 76,3 & 82,3 & 20,2 & 12,4 & 2,6 & 3,8 & 0,9 & 1,2 \\
\hline 20 & 84,2 & 87,3 & 11,4 & 7,1 & 4,4 & 3,2 & 0 & 0,9 \\
\hline 21 & 29,8 & 28,6 & 29,8 & 31,9 & 29,8 & 30,1 & 10,5 & 8,6 \\
\hline 25 & 52,6 & 60,5 & 29,8 & 22,4 & 12,3 & 11,8 & 4,4 & 5 \\
\hline 31 & 93 & 93,8 & 4,4 & 5,3 & 0,9 & 0,6 & 0,9 & 0 \\
\hline $\begin{array}{l}\text { 3. Hic } \\
\text { 5. Saq } \\
\text { 7. Le } \\
\text { 10. Le } \\
\text { 15. Le } \\
\text { 18. Le } \\
\text { 20. Le } \\
\text { 21. Le } \\
\text { 25. Le } \\
\text { 27. Le }\end{array}$ & $\begin{array}{l}\text { (Hizo) al } \\
\text { é (Sacó) } \\
\text { Me) dije ( } \\
\mathrm{Me} \text { ) hable } \\
\mathrm{Me} \text { ) insul } \\
\mathrm{Me} \text { ) ridic } \\
\mathrm{Me} \text { ) segu } \\
\mathrm{Me} \text { ) culpe } \\
\mathrm{Me} \text { ) acuse } \\
\mathrm{Me} \text { ) amen }\end{array}$ & $\begin{array}{l}\text { o para por } \\
\text { relucir alg } \\
\text { algo sólo } \\
\text { (ó) en un } \\
\text { (ó) con } \mathrm{f} \\
\text { icé (ó) o } \\
\text { (siguió) p } \\
\text { (ó) por el } \\
\text { (ó) de flir } \\
\text { cé (ó) cor }\end{array}$ & $\begin{array}{l}\text { r (me) a m } \\
\text { malo que } \\
\text { para hacer } \\
\text { no de voz } \\
\text { ases despec } \\
\text { e (se) burl } \\
\text { ra saber co } \\
\text { roblema } \\
\text { dejar o coque } \\
\text { deja rel }\end{array}$ & $\begin{array}{l}\text { i chico/a c } \\
\text { el/ella (yo } \\
\text { (me) enf } \\
\text { oostil u of } \\
\text { tivas } \\
\text { (ó) de él/ } \\
\text { n quién y } \\
\text { ear con o } \\
\text { ación }\end{array}$ & $\begin{array}{l}\text { loso/a } \\
\text { había hec } \\
\text { dar } \\
\text { nsivo } \\
\text { lla (mí) d } \\
\text { ónde estal }\end{array}$ & o en el pa & ado & \\
\hline
\end{tabular}

Por último se analizaron las relaciones entre sexismo y agresiones. Como se evidencia en la tabla 4 en la muestra total se hallaron correlaciones significativas con un índice bajo de asociación entre los sexismos y los diferentes tipos de conductas agresivas; los índices de correlación más altos asocian la agresión sexual cometida y sufrida (rho $=.9, \mathrm{n}=453, \mathrm{p}<.01$ ), la agresión verbal-emocional cometida y sufrida (rho $=.78, \mathrm{n}=453, \mathrm{p}<.01$ ), el sexismo hostil y benevolente ( $\mathrm{rho}=.64, \mathrm{n}=453, \mathrm{p}<.01$ ) y la agresión física cometida y sufrida ( $\mathrm{rho}=.53, \mathrm{n}=$ 453, p<.05). Ver Tabla 4. 
Tabla 4. Correlaciones entre sexismos y agresiones físicas, sexuales y verbal-emocionales, cometidas y sufridas, en la muestra total $(n=453)$

\begin{tabular}{|c|c|c|c|c|c|c|c|c|}
\hline & 1 & 2 & 3 & 4 & 5 & 6 & 7 & 8 \\
\hline (1) & - & & & & & & & \\
\hline (2) & $.9^{* *}$ & - & & & & & & \\
\hline (3) & $.31 * *$ & $.36^{* *}$ & - & & & & & \\
\hline (4) & $.32 * *$ & $.39 * *$ & $.78^{* *}$ & - & & & & \\
\hline (5) & $.14 * *$ & $.19^{* *}$ & $.34 * *$ & $.28^{* *}$ & - & & & \\
\hline (6) & $.17 * *$ & $.23^{* *}$ & $.29 * *$ & $.34 * *$ & $.53^{*}$ & - & & \\
\hline (7) & $.14 * *$ & $.15^{* *}$ & $.105^{*}$ & $.12 * *$ & -.005 & .08 & - & \\
\hline (8) & $.11^{*}$ & $.14^{* *}$ & $.13^{* *}$ & $.14 * *$ & .001 & .02 & $.64 * *$ & - \\
\hline \multicolumn{9}{|c|}{$* * p<.01 \quad * p<.05$} \\
\hline \multicolumn{9}{|c|}{$\begin{array}{l}\text { (1) Agresión sexual cometida } \\
\text { (2) Agresión sexual sufrida } \\
\text { (3) Agresión verbal-emocional cometida } \\
\text { (4) Agresión verbal-emocional sufrida } \\
\text { (5) Agresión física cometida } \\
\text { (6) Agresión física sufrida } \\
\text { (7) Sexismo hostil } \\
\text { (8) Sexismo benevolente } \\
\end{array}$} \\
\hline
\end{tabular}

En la muestra de hombres no hubo una correlación significativa entre el sexismo hostil y las agresiones físicas cometidas ( $\mathrm{rho}=.12, \mathrm{n}=114$ ) o las agresiones verbales-emocionales cometidas (rho $=.11, \mathrm{n}=114$ ), aunque sí se halló una baja correlación positiva entre el sexismo hostil y las agresiones sexuales cometidas ( $\mathrm{rho}=.19, \mathrm{n}=114, \mathrm{p}<.05$ ). No se obtuvo una correlación significativa entre el sexismo benevolente y las agresiones físicas ( rho $=.03$; $\mathrm{n}=114$ ) o verbales-emocionales cometidas ( $\mathrm{rho}=.01, \mathrm{n}=114)$, sin embargo se hallaron bajas correlaciones positivas entre el sexismo benevolente y las agresiones sexuales cometidas (rho $=.19, \mathrm{n}=114, \mathrm{p}<.05)$, relación que positiva y no negativa como se esperaba, y entre el sexismo hostil y las agresiones sexuales cometidas $(\mathrm{rho}=.18, \mathrm{n}=114, \mathrm{p}<.05)$. Ver Tabla 5. 
Tabla 5. Correlaciones entre sexismos y agresiones físicas, sexuales y verbal-emocionales, cometidas y sufridas, en chicos $(n=114)$

\begin{tabular}{ccccccccc}
\hline & 1 & 2 & 3 & 4 & 5 & 6 & 7 & 8 \\
\hline$(1)$ & - & & & & & & & \\
(2) & $.9 * *$ & - & & & & & & \\
(3) & $.49^{* *}$ & $.47 * *$ & - & & & & & \\
(4) & $.47^{* *}$ & $.51^{* *}$ & $.83^{* *}$ & - & & & & \\
(5) & $.33^{* *}$ & $.37^{* *}$ & $.37^{* *}$ & $.34^{* *}$ & - & & & \\
(6) & $.31^{* *}$ & $.37^{* *}$ & $.45^{* *}$ & $.48^{* *}$ & $.7 * *$ & - & & \\
(7) & $.19^{*}$ & .17 & .11 & .06 & .12 & .04 & - & \\
(8) & $.18^{*}$ & .16 & .01 & .01 & .06 & .02 & $.78^{* *}$ & - \\
\hline
\end{tabular}
$* * p<.01 * p<.05$
(1) Agresión sexual cometida
(2) Agresión sexual sufrida
(3) Agresión verbal-emocional cometida
(4) Agresión verbal-emocional sufrida
(5) Agresión física cometida
(6) Agresión física sufrida
(7) Sexismo hostil
(8) Sexismo benevolente

En cuanto a las mujeres, los resultados indican correlaciones significativas y de magnitudes importantes entre la agresión sexual cometida y sufrida ( agresión verbal-emocional cometida y sufrida ( $\mathrm{rho}=.76, \mathrm{n}=339, \mathrm{p}<.01$ ), el sexismo hostil y benevolente ( $\mathrm{rho}=.64, \mathrm{n}=339, \mathrm{p}<.01)$ y la agresión física cometida y sufrida (rho $=.49, \mathrm{n}=$ $339, \mathrm{p}<.01$ ); sin embargo, como puede observarse en la tabla 6 hay otras correlaciones significativas con índices más bajos que asocian distintos tipos de agresiones cometidas o sufridas. Por último, los índices de correlación más bajos correspondieron a las asociaciones entre el sexismo benevolente con la agresión verbal-emocional sufrida (rho $=.18, \mathrm{n}=339, \mathrm{p}<.01$ ), la agresión verbal-emocional cometida (rho $=.16, \mathrm{n}=339, \mathrm{p}<.01$ ) y la agresión sexual sufrida (rho $=.15, \mathrm{n}=339, \mathrm{p}<.05)$; mientras que el sexismo hostil mostró correlaciones con la agresión verbal-emocional cometida ( $\mathrm{rho}=.14, \mathrm{n}=339, \mathrm{p}<.01$ ), la agresión verbal-emocional 
sufrida (rho $=.13 \mathrm{n}=339, \mathrm{p}<.05)$ y la agresión sexual sufrida $(\operatorname{rho}=.13, \mathrm{n}=339, \mathrm{p}<.05)$. Ver Tabla 6.

Tabla 6. Correlaciones entre sexismos y agresiones físicas, sexuales y verbal-emocionales, cometidas y sufridas, en chicas $(n=339)$

\begin{tabular}{ccccccccc}
\hline & 1 & 2 & 3 & 4 & 5 & 6 & 7 & 8 \\
\hline$(1)$ & - & & & & & & & \\
(2) & $.91^{* *}$ & - & & & & & & \\
(3) & $.26^{* *}$ & $.32^{* *}$ & - & & & & & \\
(4) & $.27^{* *}$ & $.35^{* *}$ & $.76^{* *}$ & - & & & & \\
(5) & .09 & $.15^{* *}$ & $.33^{* *}$ & $.26^{* *}$ & - & & & \\
(6) & .09 & $.17^{* *}$ & $.23^{* *}$ & $.28^{* *}$ & $.49^{* *}$ & - & & \\
(7) & .09 & $.13^{*}$ & $.14^{* *}$ & $.13^{*}$ & -.02 & .07 & - & \\
(8) & .09 & $.15^{*}$ & $.16^{* *}$ & $.18^{* *}$ & -.02 & .04 & $.64 * *$ & - \\
\hline
\end{tabular}

$* * p<.01 * p<.05$

\begin{tabular}{l}
\hline (1) Agresión sexual cometida \\
(2) Agresión sexual sufrida \\
(3) Agresión verbal-emocional cometida \\
(4) Agresión verbal-emocional sufrida \\
(5) Agresión física cometida \\
(6) Agresión física sufrida \\
(7) Sexismo hostil \\
(8) Sexismo benevolente \\
\hline
\end{tabular}

\section{Discusión y conclusiones}

Las subescalas de sexismo utilizadas presentaron un buen índice de fiabilidad. En cuanto a las subescalas de agresiones sólo la referente a las verbales-emocionales mostraron índices aceptables; los bajos índices en las otras escalas quizá se debieron a la variabilidad tan limitada que se ha encontrado en las respuestas de los participantes o por el número tan reducido de elementos que forman parte de estas pruebas (Fernández-Fuertes et al., 2008), de cualquier forma la interpretación de los hallazgos en esos rubros requieren una mayor cautela. 
Los resultados obtenidos sobre el sexismo ambivalente hacia la mujer concuerdan con investigaciones precedentes donde los niveles de sexismo benevolente detectados son más altos que en el sexismo hostil (Expósito et al., 1998; Lameiras, Rodríguez y González, 2004; Recio et al., 2007), una posible explicación de esos puntajes es la imagen positiva de la mujer propia del sexismo benevolente que estaría legitimando, incluso entre las propias mujeres (Dardenne, Dumont y Bollier, 2007), un sistema de actitudes sexistas que sigue considerando a la mujer de forma estereotipada y limitada a ciertos roles (Expósito, Herrera, Moya y Glick, 2010; López-Sáez, Morales y Lisbona, 2008). Las fuertes correlaciones halladas entre las subescalas de sexismo hostil y benevolente sugieren la complementariedad de ambas formas de sexismo (Rodríguez, Lameiras, Carrera y Faílde, 2010).

La ausencia de diferencias significativas entre hombres y mujeres en la perpetración de agresiones físicas coincide con los hallazgos de otros estudios (Fernández-Fuertes y Fuertes, 2010; Muñoz-Rivas, Graña, O’Leary y González, 2007b) donde se indica su posible carácter bidireccional (Cáceres, 2004; Póo y Vizcarra, 2008; Straus y Ramírez, 2007). Sin embargo, dada la naturaleza de este tipo de agresiones, en especial la femenina, es necesario conocer más sobre sus precipitantes y determinar entre otras cuestiones: las emociones (Muñoz-Rivas et al., 2007a) y motivaciones para que se presenten este tipo de conductas (Fernández-Fuertes y Fuertes, 2010) o cómo es que estos actos podrían llegar a generar, en dado caso, un intercambio de actos agresivos entre sexos (Capaldi, Kim y Shortt, 2007). En ese mismo sentido la confirmación de que los hombres perpetraron con más frecuencia agresiones sexuales podría pasar a segundo plano cuando se observa la distribución de respuestas como en el ítem 17 o las respuestas de los hombres a los ítems 2, 11 ó 31 (véase tabla 2) donde presentaron pocas diferencias con relación a las mujeres. Sin duda ello implica la necesidad de contextualizar y constatar la efectiva perpetración de este tipo de conductas por parte de las mujeres o la percepción de ello por parte de los hombres (Hernández, Vidiella, Herraiz y Sancho, 2007) así como las razones o motivaciones para que las mujeres llevaran a cabo, en su caso, estos comportamientos. Mientras esto no se clarifique, la vivencia de las agresiones sexuales en los hombres y mujeres de este estudio podría estar apoyando algunos planteamientos sugeridos en trabajos previos como la naturaleza bidireccional de estas conductas (Archer, 2000), el "doble rol” de agresores/as y víctimas (Ortega, Ortega y Sánchez, 2008) o la idea de que las agresiones sexuales cometidas por mujeres pudieran estar más presentes de lo que comúnmente se ha pensado (Krahé, Waizenhöfer y Möller, 2003). 
Los resultados hallados en las agresiones verbales-emocionales concuerdan con los antecedentes que señalan a la violencia psicológica como la forma más común de violencia en universitarios (Muñoz-Rivas et al., 2007a,b; Toldos, 2005) y donde la bidireccionalidad en este tipo de agresiones también ha sido sugerida (Alonso y Castellanos, 2006). La frecuencia con que se encontró este tipo de violencia no es nada trivial, sobre todo en ítems como el 3, 5, 7, 10 ó 21 (véase tabla 3) donde hay una representación de ambos sexos muy similar en agresiones menos severas y quizá mediadas por el factor social (Muñoz-Rivas et al., 2007a). Particularmente llama la atención que ítems con un contenido muy destructivo a la autoestima de la pareja, por ejemplo el 15 ("Le insulté con frases despectivas") ó 18 ("Le ridiculicé o me burlé de él/ella delante de otros"), hayan presentado una notable frecuencia porque la violencia psicológica tiene un carácter predictor de violencia física (Blázquez, Moreno y GarcíaBaamonde, 2009; González-Ortega et al., 2008) e incluso puede ser más dañina para la salud mental de la víctima que las agresiones físicas (Blázquez, Moreno y García-Baamonde, 2010).

Existen diversas propuestas explicativas sobre las conductas agresivas en la pareja que apelan a factores culturales (Ferrer y Bosch, 2005; Torrico et al., 2005), sociales (Gover, 2004) o demográficos (Foshee et al., 2008). No obstante, en el caso específico de las agresiones cometidas por mujeres, se aduce que ellas utilizan comportamientos agresivos en defensa propia, que son más introspectivas que los hombres y más capaces de recordar episodios agresivos perpetrados por ellas mismas o que se utilizan diferentes criterios entre hombres y mujeres para definir lo que es una conducta violenta (Fernández-Fuertes y Fuertes, 2010). Sin embargo, otras de las razones tentativas radican en patrones sociales relativamente aceptados (González y Santana, 2001; Toldos, 2005) o el creciente uso de conductas asociadas al género masculino con fines instrumentales, es decir mujeres que asumen "roles masculinos" o "machistas" para perpetrar diferentes tipos de agresiones conscientes de su aceptación o tolerancia social como medio para conseguir algo (Jackson y Tinkler, 2007). Desafortunadamente, el diseño de esta investigación no permite explicar estas y otras situaciones como la asociación entre los distintos tipos de agresiones que podrían sugerir vinculaciones entre distintos tipos de violencias (Krahé, Bieneck y Möller, 2005) o que la condición de perpetrador/a de algún tipo de agresión conlleve la posibilidad de ser víctima (Fernández-Fuertes et al., 2008). 
En la literatura existente sobre violencia de género se señala una estrecha relación entre sexismos y agresiones. En el caso de las mujeres la relación negativa entre el sexismo benevolente con las agresiones sufridas por ellas ya había sido indicada en otras investigaciones (Lameiras y Rodríguez, 2003; Rodríguez, Lameiras, Carrera y Faílde, 2010), sin embargo los bajos índices de correlación obtenidos al respecto no permiten fortalecer la certeza de tal asociación. En el caso de los hombres, la demostración empírica de la relación entre sexismo y agresión cometida no ha sido muy contundente, en ese sentido las diferentes relaciones halladas en este trabajo dejan más preguntas que respuestas al no permitir corroborar la fuerte asociación atribuida al sexismo sea hostil o benevolente con la perpetración de agresiones en las relaciones de pareja. Los resultados obtenidos, sin demérito alguno de los valiosos aportes feministas en la propuesta de un nuevo marco conceptual de la prevención e intervención en la violencia contra las mujeres (Cabruja, 2004), sugerirían que el sexismo es un elemento asociado y no causal de las agresiones que al combinarse con diversos factores aumenta la probabilidad de que un hombre determinado en un ambiente determinado ejerza violencia contra la mujer (Heise, 1998).

Sin duda la violencia es un fenómeno muy complejo que requiere el conocimiento de la función específica de variables y factores de riesgo tanto en víctimas como agresores para aumentar la eficacia de planes de prevención, detección e intervención (Rey, 2008). Por esa razón los resultados derivados de este trabajo sugieren la adopción de posturas ecológicas (Flinck, Åstedt-Kurki y Paavilainen, 2008), integradoras (Alonso y Castellanos, 2006) o inclusivas (Hamel, 2009) que permitan más estudios de carácter diádico, longitudinal y cualitativo (Póo y Vizcarra, 2008) y futuras acciones de prevención e intervención que, en vez de trabajar con sólo una parte del problema, consideren la posibilidad de la interrelación entre las agresiones, su potencial naturaleza bidireccional así como el trabajo con posibles agresores o agresoras y víctimas masculinas o femeninas. Ello conllevaría el uso de marcos teóricos multifactoriales alternativos a la dicotomía agresor-víctima (Ramírez, 2002) que contemplen la posibilidad de las agresiones femeninas dentro de la pareja (Swan y Snow, 2006).

Además de la detección de actitudes sexistas contra la mujer, sería muy pertinente conocer más sobre la existencia de actitudes sexistas contra el hombre para tener una concepción integradora de este fenómeno en las relaciones hombre-mujer, porque si las mujeres han estado en desventaja o relegadas a favor de los hombres es posible que puedan albergar simultáneamente creencias que perpetúen tanto la dominación como resentimientos por esta injusta situación (Del Prado y Bustillos, 2007; Rodríguez et al., 2010); lo que significaría la 
necesidad de estudios sobre la formación de nuevas identidades masculinas y femeninas (Jackson y Tinkler, 2007) o también llamadas nuevas feminidades (Moya, Páez, Glick, Fernández y Poeschl, 2002).

Dentro de las limitaciones de esta investigación se pueden destacar: 1) el tamaño y tipo de la muestra que impide la generalización de los resultados, 2) el uso de auto-informes en el estudio de actitudes y conductas como las estudiadas en este trabajo, 3) la ausencia de ítems cualitativos que permitieran la contextualización y profundización de las respuestas de los participantes y 4) la no evaluación de la deseabilidad social de las respuestas.

\section{Agradecimientos}

Los autores desean agradecer los comentarios de los revisores anónimos, al Dr. Andrés A. Fernández-Fuertes (Universidad de Cantabria) y al Dr. Antonio Seisdedos Benito (Universidad de Salamanca) por sus observaciones a una versión previa de este artículo; a los estudiantes que participaron en esta investigación y al profesorado que hizo posible la recolección de los datos primarios.

\section{Referencias}

Alonso, J. y Castellanos, J. (2006). Por un enfoque integral de la violencia familiar. Intervención Psicosocial, 15(3), 253-274.

Alonso, P., Lobato, H. y Rodríguez L. (2007). Perfiles del agresor/a, víctima, espectadores/as y víctima/agresor-a. En V. Ferrer y E. Bosch (Comps.). Los feminismos como herramientas de cambio social (II). De la violencia contra las mujeres a la construcción del pensamiento feminista (pp. 89-99). Palma: Universitat de les Illes Balears, Sevei de Publicacions i Intercanvi Cientific.

Archer, J. (2000). Sex differences in aggression between heterosexual partners: A metaanalytic review. Psychological Bulletin, 126(5), 651-680.

Begany, J. y Milburn, M. (2002). Psychological Predictors of Sexual Harassment: Authoritarianism, Hostile Sexism, and Rape Myths. Psychology of Men and Masculinity, $3(2), 119-126$.

Blázquez, M., Moreno, J.M. y García-Baamonde, M.E. (2009). A study of psychological abuse in couple relationships in young university students. Electronic Journal of Re- 
search in Educational Phychology, 7(2), 691-714. Obtenido el 2 de enero de 2011 en

http://www.investigacion-psicopedagogica.org/revista/articulos/18/english/Art_18_337.pdf

Blázquez, M., Moreno, J. M. y García-Baamonde, M.E. (2010). Revisión teórica del maltrato psicológico en la violencia conyugal. Psicología y Salud, 20(1), 65-75.

Cabruja, T. (2004). Violencia doméstica: sexo y género en las teorías psicosociales sobre la violencia. Hacia otras propuestas de comprensión e intervención. Intervención Psicosocial, 13(2), 141-153.

Cáceres, J. (2004). Violencia física, psicológica y sexual en el ámbito de la pareja: papel del contexto. Clínica y Salud, 15(1), 33-54.

Capaldi, D.M, Kim, H.K. y Shortt, J.W. (2007). Observed initiation and reciprocity of physical aggression in young, at risk couples. Journal of Family Violence, 22(2), 101-111.

Chapleau, K., Oswald, D. y Russell, B. (2007). How ambivalent sexism toward women and men support rape myth acceptance. Sex Roles, 5, 131-136.

Dardenne, B., Dumont, M. y Bollier, T. (2007). Insidious dangers of benevolent sexism: Consequences for women's performance. Journal of Personality and Social Psychology, 93(5), 764-779.

Del Prado, M. y Bustillos, A. (2007). Benevolent sexism toward men and women: Justification of the traditional system and conventional gender roles in Spain. Sex Roles, 57, 607-614.

Expósito, F. y Herrera, M.C. (2009). Social perception of violence against women: individual and psychosocial characteristics of victims and abusers. The European Journal of Psychology Applied to Legal Context, 1(1), 123-145.

Expósito, F., Herrera, M.C., Moya, M. y Glick, P. (2010). Don’t rock the boat: women's benevolent sexism predicts fears of marital violence. Psychology of Women Quarterly, 34(1), 36-42.

Expósito, F., Moya, M., y Glick, P. (1998). Sexismo ambivalente: medición y correlatos. Revista de Psicología Social, 13, 159-169.

Expósito, F. y Moya, M. (2005). Sexismo y aceptación de la Violencia de Género en las relaciones íntimas. En J. Sobral, G. Serrano y J. Regueiro (Comps.), Psicología jurídica, de la violencia y de género (pp. 321-328). Madrid: Biblioteca Nueva.

Fernández-Fuertes, A.A., y Fuertes, A. (2010). Physical and psychological aggression in dating relationships of Spanish adolescents: Motives and consequences, Child Abuse \& Neglect, 34, 183-191. 
Fernández-Fuertes, A.A., Fuertes, A. y Orgaz, B. (2008). El CADRI en el estudio del comportamiento agresivo en las relaciones de pareja adolescentes. En J.A. González-Pineda y J.C. Núñez Pérez (Eds.), Psicología y Educación: un lugar de encuentro (pp. 1622-1630). Oviedo: Ediciones de la Universidad de Oviedo.

Fernández-Fuertes, A.A., Fuertes, A. y Pulido R. F. (2006). Evaluación de la violencia en las relaciones de pareja de los adolescentes. Validación del Conflict in Adolescent Dating Relationships Inventory (CADRI) versión española. International Journal of Clinical Health Psychology, 6, 339-358.

Ferrer, V.A. y Bosch, E. (2000). Violencia de género y misoginia: reflexiones psicosociales de un posible factor explicativo. Papeles del Psicólogo, 75, 13-19.

Ferrer, V.A. y Bosch, E. (2005). Análisis empírico de factores de riesgo en la violencia contra las mujeres en la pareja. En J. Sobral, G. Serrano y J. Regueiro (Comps.), Psicología jurídica, de la violencia y de género (pp. 255-264). Madrid: Biblioteca Nueva.

Ferrer, V.A., Bosch, E., Ramis, M.C., Torres, G., y Navarro, C. (2006). Violencia contra las mujeres en la pareja: creencias y actitudes en estudiantes universitarios/as. Psicothema, 18, 359-366.

Flinck, A., Åstedt-Kurki, P. y Paavilainen, E. (2008). Intimate partner violence as experienced by men. Journal of Psychiatric and Mental Health Nursing, 15, 322-327.

Foshee, V.A., Karriker-Jaffe, K.J., Reyes, H.L., Ennett, S.T., Suchindran, C., Bauman, K.E., y Benefield, T.S. (2008). What accounts for demographic differences in trajectories of adolescent dating violence? An examination of intrapersonal and contextual mediators. Journal of Adolescent Health, 42, 596-604.

García-Leiva, P., Palacios, M.S., Torrico, E. y Navarro, Y. (2007, mayo/junio). El sexismo ambivalente: ¿un predictor del maltrato? Boletín Electrónico de Psicología Jurídica $y$ Forense, 29. Obtenido el 26 de noviembre de 2010 en http://psicologiajuridica.org/psj210.html el.

Glick, P. y Fiske, S. (1996). The ambivalent sexism inventory: Differentiating hostile and benevolent sexism. Journal of Personality and Social Psychology, 70, 491-512.

González, R. y Santana, J.D. (2001). La violencia en parejas jóvenes. Psicothema, 13(1), 127 131.

González-Ortega, I., Echeburúa, E. y Corral, P. (2008). Variables significativas en las relaciones violentas en parejas jóvenes: una revisión. Psicología Conductual, 16(2), 207225. 
Gover, A.R. (2004). Risky lifestyles and dating violence: A theoretical test of violent victimization. Journal of Criminal Justice, 32, 171-180.

Hamel, J. (2009). Toward a gender-inclusive conception of intimate partner violence research and theory: Part 2 - New directions. International Journal of Men's Health, 8(1), 41-59.

Hattery, A.J. (2009). Intimate partner violence. Maryland: Rowman \& Littlefield Publishers.

Heise, L. (1998). Violence against women: an integrated, ecological framework. Violence against women, 4(3), 262-290.

Hernández, F., Vidiella, J., Herraiz, F., y Sancho, J.M. (2007). El papel de la violencia en el aprendizaje de las masculinidades. Revista de Educación, 342, 103-125.

Jackson, C. y Tinkler, P. (2007). 'Ladettes' and 'Modern Girls': ‘troublesome' young femininities. The Sociological Review, 55(2), 251-272.

Krahé, B., Bieneck, S. y Möller, I. (2005). Understanding gender and intimate partner violence from an international perspective. Sex Roles, 52(11-12), 807-827.

Krahé, B., Bieneck. S. y Scheinberger-Olwig, R. (2007). The role of Sexual scripts in sexual aggression and victimization. Journal of Sex Research, 44(4), 316-327.

Krahé, B., Waizenhöfer, E. y Möller, I. (2003). Women's sexual aggression against men: prevalence and predictors. Sex Roles, 49(5-6), 219-232.

Kurz, D. (1997). Violence against Women or Family Violence? Current debates and future directions. En L. O’Toole y J. Schiffman (Eds.). Gender Violence. Interdisciplinary perspectives (pp. 443-453). New York: New York University Press.

Lameiras, M. y Rodríguez, Y. (2003). Evaluación del sexismo ambivalente en estudiantes gallegos/as. Acción psicológica, 2(2), 131-136.

Lameiras, M., Rodríguez, Y. y González, M. (2004). Evolution of hostil sexism and benevolent sexism in a Spanish sample. Social Indicators Research, 66, 197-211.

López-Sáez, M., Morales, J. F. y Lisbona, A. (2008). Evolution of gender stereotypes in Spain: traits and roles. The Spanish Journal of Psychology, 11(2), 609-617.

Moya, M., Páez, D., Glick, P., Fernández, I., Poeschl, G. (2002). Sexismo, masculinidadfeminidad y factores culturales. Revista Española de Motivación y Emoción, 3, 127142.

Muñoz-Rivas, M.J, Graña, J.L., O’Leary, K.D. y González, P. (2007a). Aggression in adolescent dating relationships: Prevalence, justification, and health consequences. Journal of Adolescent Health, 40(4), 298-304. 
Muñoz-Rivas, M.J., Graña, J.L., O’Leary, K.D. y González, P. (2007b). Physical and psychological aggression in dating relationships in Spanish university students. Psicothema, 19(1), 102-107.

Murnen, S.K., Wright, C., y Kaluzny, G. (2002). If 'boys will be boys,' then girls will be victims? A meta-analytic review of the research that relates masculine ideology to sexual aggression. Sex Roles, 46, 359-375.

Ortega, R., Ortega, F.J. y Sánchez, V. (2008). Violencia sexual entre compañeros y violencia en parejas adolescentes. International Journal of Psychology and Psychological Therapy, 8(1), 63-72.

Palacios, M., Torrico, E., Millán, M., Pérez, M., Puertas, S., Moya, M. y López, J. (2005). Violencia contra las mujeres y sexismo ambivalente. En J. Sobral, G. Serrano y J. Regueiro (Comps.), Psicología jurídica, de la violencia y de género (pp. 345-357). Madrid: Biblioteca Nueva.

Pelegrín, A. y Garcés, E.J. (2004). Aproximación teórico-descriptiva de la violencia de género: propuestas para la prevención. Apuntes de Psicología, 22(3), 353-373.

Póo, A.M., y Vizcarra, M.B. (2008) Violencia de pareja en jóvenes universitarios. Terapia psicológica, 26(1), 81-88.

Pozo, C., Alonso, E., Hernández, S. y Mellado, M. (2005). Actitudes de género, creencias y sexismo ambivalente como predictores de la discriminación y violencia contra las mujeres. En J. Sobral, G. Serrano y J. Regueiro (Comps.), Psicología jurídica, de la violencia y de género (pp. 271-278). Madrid: Biblioteca Nueva.

Pozo, C., Martos M.J. y Alonso, E. (2010). ¿Do Students in Secundary Education Manifest Sexist Attitudes? Electronic Journal of Research in Educational Psychology, 8(2), 541-560. Obtenido el 1 de octubre de 2010 en http://www.investigacionpsicopedagogica.org/revista/articulos/21/english/Art_21_440.pdf

Ramírez, J.C. (2002). Pensando la violencia que ejercen los hombres contra sus parejas: problemas y cuestionamientos. Papeles de Población, 31, 219-241.

Recio, P., Cuadrado, I. y Ramos, E. (2007). Propiedades psicométricas de la Escala de Detección de Sexismo en Adolescentes (DSA). Psicothema, 19(3), 522-528.

Rey, C.A. (2008). Prevalencia, factores de riesgo y problemáticas asociadas con la violencia en el noviazgo Una revisión de la literatura. Avances en Psicología Latinoamericana, 26(2), 227-241. 
Rodríguez, Y., Lameiras, M., Carrera, M.V y Faílde, J.M. (2010). "Evaluación de las actitudes sexistas en estudiantes españoles/as de educación secundaria obligatoria". Psychologia: avances de la disciplina, 4(1), 11-24.

Ruiz, J., Expósito, F. y Bonache, H. (2010). Adolescent witnesses in cases of teen dating violence: an analysis of peer responses. The European journal of psychology applied to legal context, 2(1), 37-53.

Santana, M.C., Raj, A., Decker, M.R., La Marche, A. y Silverman, J.G. (2006). Masculine gender roles associated with increased sexual risk and intimate partner violence perpetration among young adult men. Journal of urban health, 83(4), 575-585.

Straus, M.A. y Ramirez, I. (2007). Gender symmetry in prevalence, severity, and chronicity of physical aggression against dating partners by university students in Mexico and USA. Aggressive Behavior, 33(4), 281-290.

Swan, S.C. y Snow, D.L. (2006). The development of a theory of women's use of violence in intimate relationships. Violence Against Women, 12(11), 1026-1045.

Toldos, M.P. (2005). Sex and age differences in self-estimated physical, verbal, and indirect aggression in Spanish adolescents. Aggressive Behavior, 31, 13-23.

Torrico, E., Palacios, M., Millán, M., Santín, C., Navarro, Y., García-Leiva, P., López-López, M. y Pérez, M. (2005). Maltrato y abuso en estudiantes universitarios. En J. Sobral, G. Serrano y J. Regueiro (Comps.), Psicología jurídica, de la violencia y de género (pp. 215-227). Madrid: Biblioteca Nueva.

Wolfe, D.A., Scott, K., Reitzel-Jaffe, D., Wekerle, C., Grasley, C. y Pittman, A.L. (2001). Development and validation of the conflict in adolescent dating relationships inventory. Psychological Assessment, 13, 277-293. 